\title{
Development of a simulation model of an integrated multi- energy system based on the energy hub concept
}

\author{
Nikolai Voropai 1,2,*, Dmitry Gerasimov ${ }^{2}$, Ekaterina Serdyukova ${ }^{1,2}$, Konstantin Suslov ${ }^{2}$. \\ ${ }^{1}$ Melentiev Energy Systems Institute, Siberian Branch of the Russian Academy of Sciences, Irkutsk, Russia \\ 2 Irkutsk National Research Technical University, Irkutsk, Russia
}

\begin{abstract}
Transformation of energy systems under the impact of internal and external factors remarkably strengthens the technological integration of those systems and supports development of integrated multienergy systems. The paper discusses the problems of modeling the integrated energy systems and proposes an approach to construction of a simulation model using the Matlab/Simulink software and the energy hub concept.
\end{abstract}

Key words: integrated multi-energy system, energy hub, simulation model.

\section{Introduction}

Energy systems in the latest decades (electric power, heat, gas, oil and oil products supply systems, etc.) undergo radical transformations of their structure and properties under the impact of internal and external factors. Strengthening of the technological integration of those systems and creation of integrated multi-energy systems is the major trend of such integration. For example, until recently the integration of power, heat and gas supply systems regarding interdependence of their regimes operation and development was implemented mainly at gas-fired cogeneration plants $(\mathrm{CP})$ producing power and heat, but situation radically changed after emergence of technologies and economic mechanisms that allowed their alternative use by consumers, that is, use of different technologies for the same objectives, for example, use of either heat from cogeneration plants or electric heating, electric or gas furnaces, and some others. Operating conditions of electric, heat and gas networks happened to be interrelated accordingly. Effect of technological integration of those systems shall also be considered during justification of their development [1, 2 et al.].

Tendency towards technological integration of energy supply systems gave birth to the notion of an energy hub $[2,3]$, that implies an integrated facility with multiple inputs and outputs, that represent different types of energy, and support of the internal functions of that facility, i.e., transmission, storage and conversion of different forms of energy. It should be noted [4], that the energy hub concept can be applied in a wide range, i.e., from representation of an individual element converting several kinds of energy to a building or a part of the city.

Tendency towards strengthening the technological integration of energy supply systems provided incentives for studies on modeling the integrated multi-energy systems, for investigation and control of their operating conditions and development. This fact gave rise to development of basically different directions in modeling the integrated multi-energy systems: first, using conventional interrelated mathematical models [5, 6 et al.] and, second, using the energy hub concept [7, 8 et al.].

The present paper discusses potentials of modeling the integrated multi-energy systems based on the energy hub concept. The following section briefly discusses the basic provisions of this concept of modeling, elucidates its advantages and challenges. Further we propose directions for development of the considered approach with account of capabilities of the Matlab/Simulink software.

\section{Main provisions of the energy hub modeling}

Refs. [7, 9, 10] present in a general form a system of linear algebraic equations for converting some forms of energy into other ones, equations that relate input variables of the energy hub to its output variables. Both variables represent different forms of energy.

$$
\left(\begin{array}{c}
L_{\alpha} \\
L_{\beta} \\
\mathrm{M} \\
L_{\gamma}
\end{array}\right)=\left(\begin{array}{cccc}
C_{\alpha \alpha} & C_{\beta \alpha} & \mathrm{L} & C_{\gamma \alpha} \\
C_{\alpha \beta} & C_{\beta \beta} & \mathrm{L} & C_{\gamma \beta} \\
\mathrm{M} & \mathrm{M} & \mathrm{O} & \mathrm{M} \\
C_{\alpha \gamma} & C_{\beta \gamma} & \mathrm{L} & C_{\gamma \gamma}
\end{array}\right)\left(\begin{array}{c}
E_{\alpha} \\
E_{\beta} \\
\mathrm{M} \\
E_{\gamma}
\end{array}\right)
$$

or

$$
L=C \cdot E
$$

Energy in the input and output ports is represented by vector-columns $E=\left[E_{a}, E_{\beta}, \ldots, E_{\gamma}\right]$ и $L=\left[L_{a}, L_{\beta}, \ldots, L_{\gamma}\right], C$ is a matrix of direct relations, that describes conversion

\footnotetext{
* Corresponding author: voropai@isem.irk.ru
} 
of energy forms from input to output. Each member of the matrix relates one specific input to a certain output.

In case of solving the inverse problem, a matrix of inverse conversions is introduced

$$
\left(\begin{array}{c}
E_{\alpha} \\
\mathrm{M} \\
E_{\gamma}
\end{array}\right)=\left(\begin{array}{cc}
d_{\alpha \alpha} \mathrm{L} & d_{\gamma \alpha} \\
\mathrm{MO} & \mathrm{M} \\
d_{\alpha \gamma} \mathrm{L} & d_{\gamma \gamma}
\end{array}\right)\left(\begin{array}{c}
L_{\alpha} \\
\mathrm{M} \\
L_{\gamma}
\end{array}\right)
$$

Relations between coefficients of inverse and direct transformations have a unique form:

$$
d_{\beta \alpha}=\left\{\begin{array}{l}
c_{\alpha \beta}^{-1} \text { if } c_{\alpha \beta} \neq 0 . \\
0 \text { else }
\end{array}\right.
$$

Should there be $N$ output ports and one input port, the energy through each output channel would be distributed following the equation

$$
E_{i m}=\sum_{n=1}^{N} D_{i m} L_{i n}
$$

Recent studies, primarily thesis [11, 12], provide formalized statements of specific problems of integrated multi-energy systems, methods and results of their solution on the base of linear models of the energy hub. The main prerequisites for developing those models were described above. Consideration was also given to the problems of computation and optimization of load flows in the integrated electric, heat and gas networks, to reliability of power supply for consumers, to optimization of daily curves during their dispatching, to optimization of development of the integrated multienergy systems, and to some others $[2,4,5,7-10$ et. al.].

Studies undertaken demonstrated potentials of the considered approach and at the same time revealed the problems of its application. Merit of the approach lies in unified description of functions of the energy hub by separate channels of the energy forms transmission, distribution, conversion, and storage. But even a linear model of the energy hub faces the problems of determining the coefficients of the conversion matrix. In reality these factors are often dependent on the output variables, may have complex internal structure, and contain non-linearity. Moreover, presented models of the energy hub allow solution of only stationary problems while studying the integrated multi-energy systems. Dynamic problems have not yet been considered using the energy hub concept, though their urgency raises no doubt, and, according to [11], this trend will be priority one in the future.

It should also be noted that dynamic problems have not yet been considered using the conventional mathematical models of integrated energy systems. One of the most systematic efforts in this respect was made in [13] where a mathematical apparatus of the theory of singular disturbances (small parameters) was used for formalizing the representation of different energy systems forming an integrated multi-energy system.
The described problems of modeling an energy hub necessitate the search for other approaches to their solution. Section to follow considers some of those potentials.

\section{Modeling an integrated multi-energy system using Matlab/Simulink capabilities}

Construction of a simulation model of an integrated multi-energy system is based on the following statements:

- A simulation model of an integrated multi-energy system is constructed using models of elements in conformity with topology of connection schemes (equivalent schemes) of individual energy systems with account of their relations in the integrated system;

- Present-day versions of Matlab/Simulink software have a large library of standard blocks for simulating different technological systems: electric power, hydraulic, pneumatic ones, and others. Those blocks were standardized using transfer functions and on that base they implement the models of elements of the considered technical systems for studying the dynamic processes. Models of dynamic elements of the system, subject to appropriate assumptions, can be converted into static models for forming the appropriate static models for studying the steady-state conditions of an integrated multi-energy system;

- A library of the elements' models in the Matlab/Simulink software does not contain models of elements converting some forms of energy into other ones, and elements having several inputs and/or outputs. Such elements include, for example, a cogeneration plant with gas as input, and eclectic power and heat as output. At the level of consumers such elements of an integrated system have the function of energy conversion, for example, conversion of electric energy into thermal energy in case of using the electric heaters. Models of such elements were developed in addition to the library of simple elements using Matlab/Simulink (as a library of additional models) on the base of the energy hub concept. These models also implement such functions as energy storage and summation of the energy flows;

- Mention should be made of two forms of the energy conversion elements: elements that change characteristics of the energy channel without converting one energy form into the other one (transformers, heat exchangers, etc.) and elements that convert one energy form into the other one (eclectic heaters, gas turbine units, etc.). Some forms of energy of an integrated multienergy system have different measurement units. Joule $(\mathrm{J}, \mathrm{W} \cdot \mathrm{s})$ is taken to be a basic unit of measurements. Blocks for reducing different units to the basic one were implemented using Matlab/Simulink.

\section{Eelements of the energy hub modeling technologies}

Figure 1 presents a generalized functional structure of the simulation model of the energy hub that was developed using Matlab/Simulink [14] capabilities. This 
structural scheme presents three energy supply channels: 1 - electric power; 2 - heat; 3 - gas. The model implements the functions of transformation, conversion and storage of energy, and an additional summation function whose concept is understandable from Figure 1.

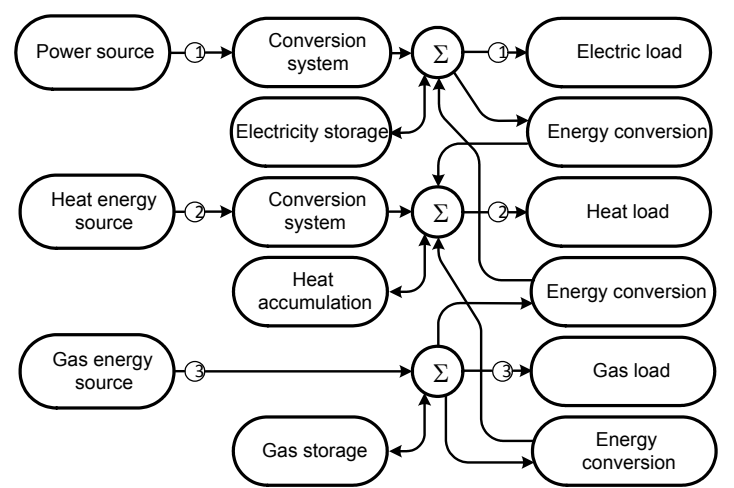

Fig. 1. Generalized functional structure of the simulation model of the energy hub (channels: 1 - electric power supply; 2 - heat supply; 3 - gas supply).

Figure 2 gives an expanded functional scheme of a part of the generalized functional structure of a simulation model of the energy hub for the power supply channel. Here 1, 6 are blocks of direct and inverse transformation of state variables; 2,4 are blocks of electricity transfer; 3 is a block for modeling the transformer substation; 5 is a block for modeling the energy storage device.

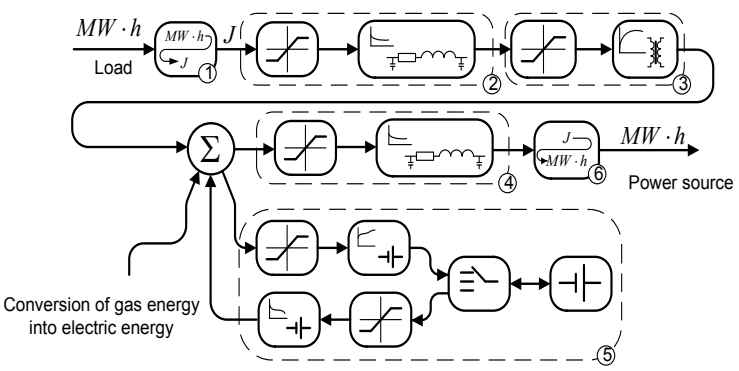

Fig. 2. A functional scheme of a power supply channel of a simulation model of the energy hub

This functional scheme of a power supply channel takes into account peculiarities of simulation modeling using Simulink/MatLab system, particularly, rules for propagation and conversion of signals in this system.

\section{Implementation of a simulation model of an integrated multi-energy system Formatting the text}

A general approach to constructing a simulation model of an integrated multi-energy system and to solution of different problems with its help can be represented as follows $[14,15]$.

a) Input information on the studied integrated multienergy system is set in the form of matrices of parameters of relations between electric and pipeline networks (their electric and hydraulic resistances) with their reference of those relations to specific numbers of nodes, as well as vectors of nodes parameters (loads, power generation, energy storage systems, etc.). b) Two libraries of the models of elements are used for constructing a simulation model of an integrated system: a library of standard models available in Matlab/Simulink and a library of specially developed additional models.

c) An algorithm for constructing a simulation model of an integrated energy system subsequently selects (depending on the element type) the required model of that element either from the library of standard models or from the library of additional models by analysing the topology of every energy system, and attaches it to the required node of the constructed system.

d) Developed additional models containing several inputs and several outputs represent generating (feeding) nodes (CP) or load nodes that include interrelated consumption of several alternative types of energy. Those nodes integrate individual energy systems into a single integrated system.

e) Solution of some problems requires information additional to that mentioned in paragraph (a). Such problems require appropriate modification of the constructed simulation model.

Figure 3 gives an example of an integrated scheme based on two energy supply channels. A black line here denotes a channel of a heat network; grey one denotes a channel of an electric network. A square denotes hub location.

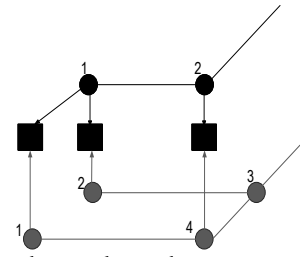

Fig. 3. An integrated scheme based on two energy supply channels

As is seen from Figure 3, two energy channels go to one consumer. Should storage systems and systems for converting the electric energy into thermal one be used in those nodes of an integrated scheme, energy supply modes would be computed using a computation algorithm applied in the energy hub concept.

An electric system and a heat supply system can be modeled using standard models from Simulink library. According to the above said, specially developed blocks are used inside the energy hub. Figure 4 shows an integrated scheme of energy supply systems and an energy hub with two energy supply channels.

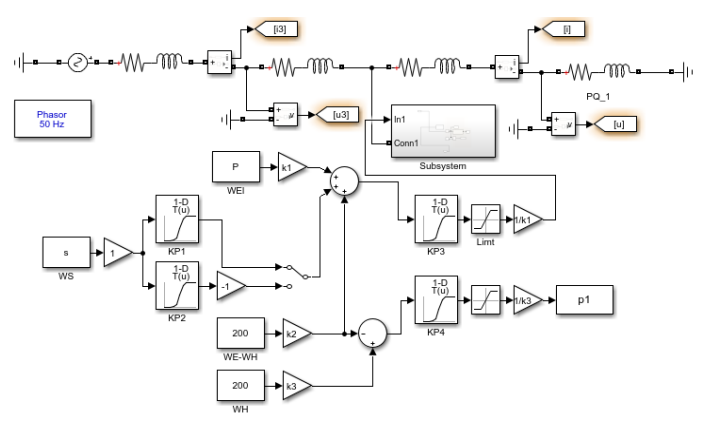

Fig. 4. Realization of an integrated scheme with two energy supply channels in Matlab/Simulink 
An energy supply system is simulated using standard blocks from the basic library of Sim Power Systems.

WEI, WS blocks represent energy consumption and storage via an electric supply channel; WH is energy consumption through a heat supply channel. KP1-KP4 are blocks taking into account efficiency of an energy converter. WE-WH are electric energy converted into heat energy.

Matlab system contains an object-oriented programming language that allows automatic construction of complex simulation models of energy systems with different energy supply channels with account of the main provisions of the energy hub concept. Figure $5 \mathrm{p}$ resents a simplified block-diagram of an algorithm for constructing a simulation model of an integrated multi-energy system using above provisions on the example of an integrated system of electricity, heat and gas supply.

Real topology of individual systems of an integrated multi-energy system may not be congruent. But there are nodes that are common for all the individual systems that integrate them. Such integrating nodes (elements) include either nodes for energy production and conversion (e.g., CP with gas as input, electricity and heat as output), or complex consumers that can use different forms of energy for the same production objectives. Those elements are modeled based on the energy hub concept and are stored in the library of additional models. When developing an algorithm for constructing a simulation model of an integrated system, such elements are purposefully fixed as ones integrating separate energy systems into an integrated multi-energy system.

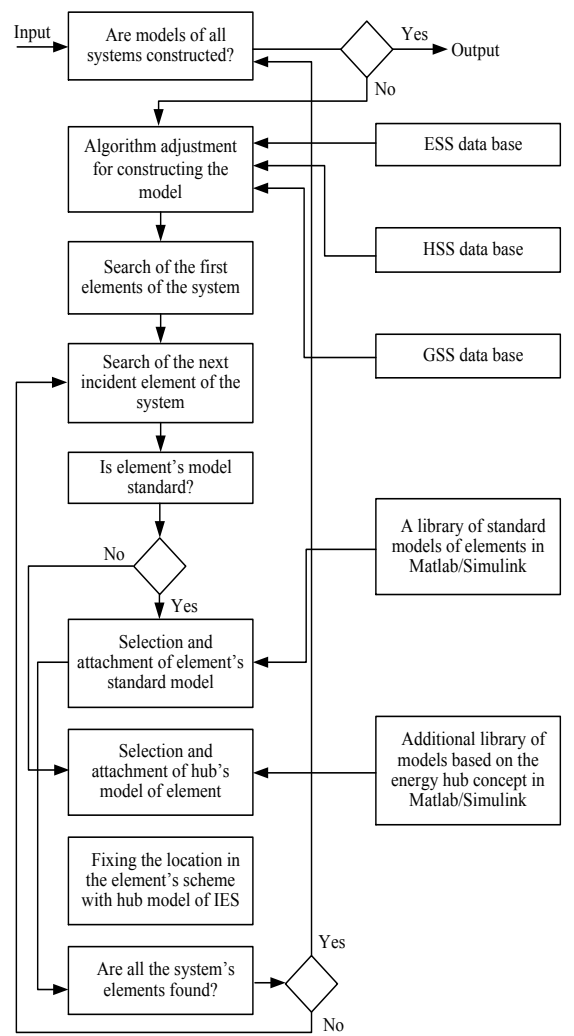

Fig. 5. Generalized block-diagram of an algorithm for constructing a simulation model of an integrated multi-energy system
Remaining (standard) elements of an individual energy system are responsible for its topology irrespective of other systems of an integrated multienergy system.

\section{Conclusion}

A concept of an energy hub is a rather practical approach to solving the problems of integrated multienergy systems, but its implementation faces certain difficulties. To overcome those difficulties the authors are developing an alternative approach to construction of a simulation model of the integrated system using Matlab/Simulink software capabilities. The paper discusses the main principles of constructing such a simulation model.

The reported study was funded by RFBR, project number 19-38-90198

\section{References}

1. Voropai NI, Stennikov V.A. Integrated intelligent energy systems // Izvestiya RAN. Energy, 2014, No. 1, pp. 64-73. (in Russian).

2. Arnold M., Andersson G. Decomposed electricity and natural gas optimal power flow // 16th Power System Computation Conference, Glasgow, Scottland, UK, July 26 - 30, 2008, 6 p.

3. Geidl M., Koeppel G., Favre-Perrod P., Andersson G., e. a. Energy Hubs for the future: A powerfull approach for next-generation energy systems // IEEE Power and Energy Magazine, 2007, Vol. 5, No. 1, pp. $24-30$.

4. Koeppel G., Andersson G. Reliability modeling of multi-carrier energy systems // Energy, 2009, Vol. 34, No. 3, pp. $235-244$.

5. Chaudry M., Jenkins N., Strbac G. Multi-time period combined gas and electricity network optimization // Electric Power System Research, 2008, Vol. 78, No. 5 , pp. $1265-1279$.

6. Voropai N.I., Stennikov V.A., Barakhtenko E.A., e. a. A model for control of a steady-state of intelligent integrated energy system // Energy Systems Research, 2018, Vol. 1, No. 1, pp. 57 - 66.

7. Geidl M. Optimal power flow of multiple energy carriers // IEEE Transactions on Power Systems, 2007, Vol. 22, No. 1, pp. 145 - 155.

8. Almassalkhi M., Hiskens I. Optimization framework for the analysis of large-scale networks of energy hubs // 17th Power System Computation Conference, Stockholm, Sweden, August $22-26$, 2011, 7 p.

9. Geidl M., Andersson G. Optimal coupling of energy infrastructures // 2007 IEEE Lausanne Power Tech, Lausanne, Switzerland, July 17 - 21, 2007, 6 p. 
10. Zhang X., Shahidehpour M., Alabdulwahab A., Abusorrah A. Optimal expansion planning of energy hub with multiple energy infrastructures // IEEE Transactions on Smart Grid, 2015, Vol. 6, No. 5, pp. $2302-2311$.

11. Geidl M. Integrated modeling and optimization of multi-carrier energy systems / PhD Dissertation. Swiss Federal Institute of Technology, Zurich, Switzerland, 2007, 125 p.

12. Koeppel G.A. Reliability considerations of future energy systems: Multi-carrier systems and the effect of energy storage / PhD Dissertation. Swiss Federal Institute of Technology, Zurich, Switzerland, 2007, $139 \mathrm{p}$.

13. Fu Shen, Ping Ju, Shahidehpour M., e. a. Singular perturbation for the dynamic modeling of integrated energy systems // IEEE Transactions on Power Systems, 2020, Vol. 35, No. 3, pp. 1718 - 1728.

14. Voropai N., Gerasimov D., Ukolova Ek., Suslov K., e. a. Simulation approach to integrated energy systems study based on the energy hub concept // 2019 IEEE Power Tech, Milan, Italy, June 23 - 27, 2019,5 p.

15. Voropai N.I., Gerasimov D.O., Serdyukova E.V., Suslov K.V. Designing a simulation model of integrated multi-carrier energy system using the energy hub concept // Methodological Problems of Large Energy Systems Reliability Study. Int. Conf. Proceedings. Kasan, Russia, September 21 - 25, 2020, Issue 2, pp. 333 - 342. (in Russian). 